\title{
Fuzzification and Defuzzification in Fuzzy AHP for Supporting Human Decision Making
}

\author{
Toshiyuki YAMASHITA*
}

\begin{abstract}
Analytic Hierarchy Process (AHP) is one of the most popular tools for supporting human decision making, and several fuzzy extensions of AHP have been proposed. The present paper discusses psychological effects of both fuzzy rating and crisp feedback of the results in fuzzy AHP. Fuzzy rating in fuzzy AHP could incorporate the fuzziness of a person's feelings in his/her decision making. Crisp feedback, which exaggerates the superiority of only one alternative or the differences among the alternatives, could help a person make his/her decision, especially when being deeply puzzled about his/her choice. The paper suggests that using both fuzzification and defuzzification in fuzzy AHP is very important for supporting human decision making.
\end{abstract}

Keywords : AHP, fuzzy AHP, decision making, fuzzy rating, fuzzy numbers

\section{Introduction}

Since people often rely on their feelings in choosing and deciding their behavior in everyday life, appropriate supporting tools for people to reach satisfying goals are very important ${ }^{[1],[2]}$. One of the most popular tools is Analytic Hierarchy Process (AHP) by

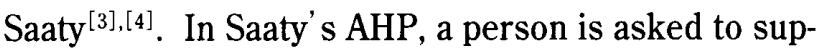
ply ratios for each pairwise comparison between alternatives $A_{1}, A_{2}, \ldots, A_{m}$ and also between the criteria $C_{1}, C_{2}, \ldots, C_{n}$. However, it seems to be more natural for people to feel that the ratio is approximately 5 to 1 or that the ratio is between 4 to 1 and 6 to 1 rather than for people to feel that the ratio is exactly 5 to 1 . Therefore, Saaty's AHP has been extended ${ }^{[5],[6]}$ by using fuzzy theory ${ }^{[7]}$, which provides a mathematical method able to deal with feelings and cognitive processes which are too imprecise to be dealt with by classical mathematical techniques.

Some fuzzy AHPs have used fuzzy ratings, which can incorporate the fuzziness and vagueness of a person's feelings in his/her decision making ${ }^{[8],[9],[10]}$. However, the indeterminacy about the final fuzzy weights might make it more difficult for the decision maker to understand the suggestion of the results and to choose a specific alternative. Yamashita and Yonezawa ${ }^{[11]}$ revealed that the decision maker often

* Graduate School of Engineering, Tokyo Metropolitan University wants the result which exaggerates the ascendancy of only one alternative.

The present paper describes that in order to use fuzzy AHP effectively, both the fuzzy rating which can incorporate a person's vague feelings and the defuzzification of the fuzzy results which can give him/her a crisp feedback are very useful for supporting his/her decision making ${ }^{[11]}$. Moreover, the paper describes that in order to defuzzificate the final fuzzy weights from fuzzy AHP and to exaggerate the superiority of only one alternative, it is very effective to apply Thurstone' $\mathbf{s}^{[12],[13]}$ paired comparison method to the fuzzy AHP results ${ }^{[14]}$. That is, the paper presents our method in which people can represent their fuzziness and vagueness in their feelings by fuzzy ratings and can get the results of fuzzy AHP as the crisp numbers.

\section{Fuzzification and Defuzzifi- cation in Fuzzy AHP}

In this chapter, fuzzy rating, Saaty's AHP, fuzzy AHP by Buckley, and Thurstone's paired comparison method will be explained in this order. All these techniques are used by the proposed approach.

\subsection{Fuzzy Rating}

Feelings and thoughts, on which people often rely in choosing and deciding their behavior in everyday life, are vague and fuzzy ${ }^{[1]}$. Therefore, incorporating 
a person's vagueness and fuzziness is very important for supporting him/her to reach satisfying goals in his/her decision making ${ }^{[2]}$. One of the most popular methods for incorporating people's fuzziness is the fuzzy rating method which takes account of his/her fuzziness by allowing ranges of scores.

For example, Hesketh et al. ${ }^{[8],[9],[10]}$ developed a computerized fuzzy graphic rating scale based on fuzzy set theory ${ }^{[7]}$. The computerized fuzzy graphic rating scale involves an application of the semantic differential method ${ }^{[15]}$, and they used it for their career counseling and guidance. In the computerized fuzzy graphic rating scale, a person not only indicates his/her most preferred point on a scale defined by bipolar anchors but also indicates how far to the left or right of the most preferred point he/she is prepared to extend his/her ratings, as shown in Figure 1. The fuzzy extensions on either side of the preferred point can be asymmetrical if he/she wishes. $\mathrm{He} / \mathrm{she}$ is also able to give "unfuzzy" responses simply by not providing extensions on either or both sides of the most preferred point. In the computerized fuzzy graphic rating scale, a triangular membership function is constructed by the three numbers which reflect the preferred point, left extension, and right extension, as shown in Figure 1.

What type of occupation would you like?

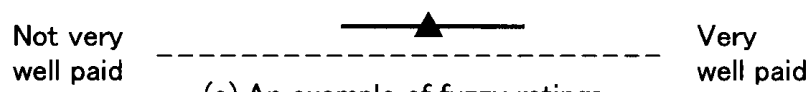

(a) An example of fuzzy ratings well paid

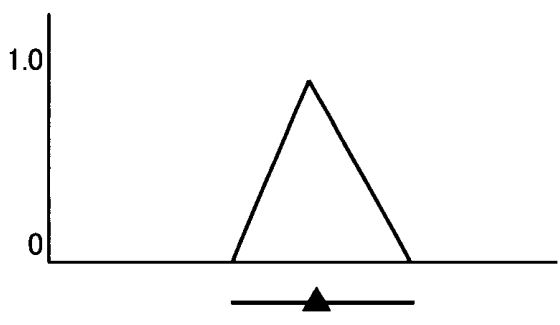

(b) Membership function

Figure 1 An example of fuzzy ratings and its membership function in the computerized fuzzy graphic rating scale ${ }^{[8],[9],[10]}$.

\subsection{Saaty's AHP}

Suppose we wish to derive relative weights for a set of $m$ alternatives according to $n$ criteria. Saaty's AHP assumes a hierarchical structure. For each criterion $C_{k}$ in a hierarchy, a person is asked to supply ratios $a_{i j}$ for each pairwise comparison between alternatives $A_{1}$, $A_{2}, \ldots, A_{m}$. The ratio $a_{i j}$ indicates the strength with which $A_{i}$ dominates $A_{j}$ for the person. The numbers for the ratios are usually taken from the set $\{1,2, \ldots$, 9\}. In Saaty's scale, $1,3,5,7$, and 9 mean "equal importance," "weak importance of one over another," "essential or strong importance," "demonstrated importance," and "absolute importance," respectively. $2,4,6$, and 8 mean intermediate values between the two adjacent judgments. If $a_{i j}$ is equal to $7 / 1$, then $a_{j i}$ is taken as $1 / 7$. That is, $a_{j i}=a_{i j}{ }^{-1}$ and $a_{i i}=1$ for all $i$. Then we obtain a positive reciprocal matrix $A_{(k)}$ for each criterion $C_{k}$.

The matrix $A_{(k)}$ obtained from a person's paired comparisons dose not satisfy the property $a_{i j} a_{j k}=a_{i k}$, and $A_{(k)}$ usually has $m$ eigenvalues and eigenvectors. Therefore, let $\lambda_{\max }$ be the largest eigenvalue of $A_{(k)}$ and $\boldsymbol{w}_{(k)}{ }^{\prime}=\left[w_{1 k}, w_{2 k}, \cdots, w_{m k}\right]$ the associated normalized eigenvector. Each element of the eigenvector $w_{(k)}, k=1,2, \ldots, n$, is the estimate of the weight of each alternative for each criterion.

We also obtain a positive reciprocal matrix $C$ for the pairwise comparisons of the criteria and get the estimates of the weights as the elements of the normalized eigenvector $c^{\prime}=\left[c_{1}, c_{2}, \cdots, c_{n}\right]$. The final weight for alternative $A_{j}$ is

$$
f_{j}=w_{j 1} \times c_{1}+w_{j 2} \times c_{2}+\ldots+w_{j n} \times c_{n} .
$$

\subsection{Fuzzy AHP}

We use the fuzzy AHP by Buckley ${ }^{[6]}$ for its simple treatment. It needs only geometric means for computing fuzzy weights. In Buckley's fuzzy AHP, the numbers of ratios are trapezoidal fuzzy numbers which are described by $(\alpha / \beta, \gamma / \delta)$ where $0<\alpha \leq \beta \leq \gamma \leq \delta$, as shown in Figure 2.

If a person believes that alternative $A_{i}$ is more important than $A_{j}$, then the fuzzy ratio $\bar{a}_{i j}=\left(\alpha_{i j} / \beta_{i j}, \gamma_{i j} /\right.$ $\left.\delta_{i j}\right)$ has $\alpha, \beta, \gamma, \delta \in\{1,2, \ldots, 9\}$ and $\bar{a}_{j i}$ is taken as $\bar{a}_{i j}{ }^{-1}$ $=\left(\delta_{i j}{ }^{-1} / \gamma_{i j}{ }^{-1}, \beta_{i j}{ }^{-1} / \alpha_{i j}{ }^{-1}\right)$. If two of the numbers $\alpha, \beta$, or $\beta, \gamma$, or $\gamma, \delta$ are equal in a fuzzy number $\bar{a}_{i j}$, then the corresponding line segment does not exist. For example, $(2 / 3,3 / 4)$ is a triangle over interval $[2,4]$ 
and $(3 / 3,3 / 5)$ consists of both a straight line segment from $(3,0)$ to $(3,1)$ and a straight line segment from $(3,1)$ to $(5,0)$. Any real number $n$ is equal to $(n / n, n / n)$. Therefore, a person feels that $A_{i}$ and $A_{j}$ are equally important, then $\bar{a}_{i j}=(1 / 1,1 / 1)$. Let $\bar{A}$ be the $m \times m$ fuzzy matrix of all paired comparisons for the alternatives $A_{1}, A_{2}, \ldots, A_{m}$. The elements in $\bar{A}$ are $\bar{a}_{i j}$ where $\bar{a}_{i j}=\bar{a}_{j i}{ }^{-1}$ and $\bar{a}_{i i}=(1 / 1,1 / 1)$ for all $i . \bar{A}$ is called a fuzzy positive reciprocal matrix.

In the fuzzy AHP, if we obtain a fuzzy positive reciprocal matrix $\overline{\boldsymbol{A}}_{(k)}$ of pairwise comparisons for each criterion $C_{k}$ and a fuzzy positive reciprocal matrix $\bar{C}$ for the pairwise comparisons of the criteria, then we can obtain the final fuzzy weight of the alternatives $A_{j}$ by the equation:

$$
\begin{aligned}
\bar{f}_{j}= & \left(\bar{w}_{j 1} \odot \bar{c}_{1}\right) \oplus\left(\bar{w}_{j 2} \odot \bar{c}_{2}\right) \oplus \cdots \\
& \oplus\left(\bar{w}_{j k} \odot \overline{\mathcal{c}}_{k}\right)
\end{aligned}
$$

where $\oplus$ and $\odot$ mean the standard fuzzy addition and multiplication for fuzzy numbers.

However, since Saaty's largest eigenvalue procedure for determining the weights is not readily extended to fuzzy matrices, Buckley ${ }^{[6]}$ proposed the following method. Now consider a fuzzy positive reciprocal matrix $\overline{\boldsymbol{A}}=\left[\bar{a}_{i j}\right]$ where $\bar{a}_{i j}=\left(\alpha_{i j} / \beta_{i j}, \gamma_{i j} / \delta_{i j}\right)$. We

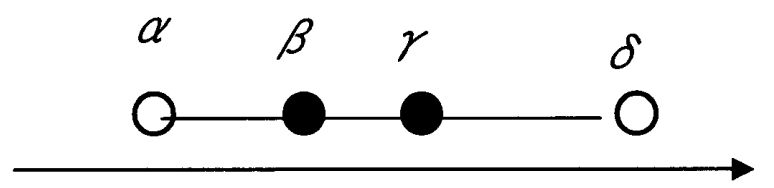

(a) An example of fuzzy ratings

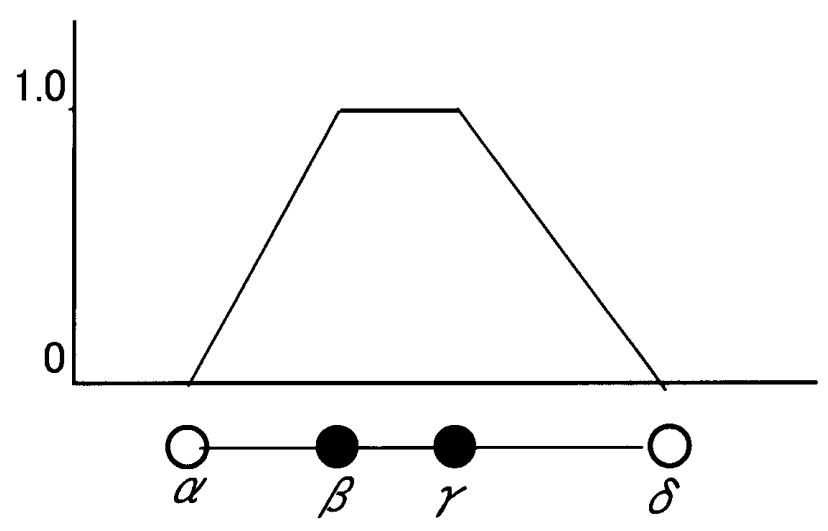

(b) Membership function

Figure 2 An example of fuzzy ratings and its membership function in the fuzzy AHP ${ }^{[6]}$. will determine the membership function $\mu_{i}$ for $w_{i}$. Let

$$
\begin{aligned}
& f_{i}(y)=\left[\prod_{j=1}^{m}\left(\left(\beta_{i j}-\alpha_{i j}\right) y+\alpha_{i j}\right)\right]^{1 / m} \\
& g_{i}(y)=\left[\prod_{j=1}^{m}\left(\left(\gamma_{i j}-\delta_{i j}\right) y+\delta_{i j}\right)\right]^{1 / m}
\end{aligned}
$$

for $0 \leqq y \leqq 1$. Define

$$
\alpha_{i}=\left[\prod_{j=1}^{m} \alpha_{i j}\right]^{1 / m}
$$

and

$$
\alpha=\sum_{i=1}^{m} \alpha_{i}
$$

Similarly, define $\beta_{i}, \beta, \gamma_{i}, \gamma, \delta_{i}$ and $\delta$. Finally, let

$$
\begin{aligned}
& f(y)=\sum_{i=1}^{m} f_{i}(y) \\
& g(y)=\sum_{i=1}^{m} g_{i}(y) .
\end{aligned}
$$

The fuzzy weights $\bar{w}_{i}$ are determined by $\left(\alpha_{i} \delta^{-1}\right)$ $\left.\beta_{i} \gamma^{-1}, \gamma_{i} \beta^{-1} / \delta_{i} \alpha^{-1}\right)$ where the graph of $\mu_{i}$ is zero to the left of $\alpha_{i} \delta^{-1}, x=f_{i}(y) / g(y)$ on the interval $\left[\alpha_{i} \delta^{-1}, \beta_{i} \gamma^{-1}\right]$, a horizontal line from $\left(\beta_{i} \gamma^{-1}, 1\right)$ to $\left(\gamma_{i} \beta^{-1}, 1\right), x=$ $g_{i}(y) / f(y)$ on the interval $\left[\gamma_{i} \beta^{-1}, \delta_{i} \alpha^{-1}\right]$, and zero to the right of $\delta_{i} \alpha^{-1}$.

\subsection{Paired comparison method}

Thurstone's model of paired comparisons ${ }^{[12],[13]}$ is as follows. A person's sensations $X_{i}$ for stimulus $A_{i}$ $(i=1,2, \cdots, n)$ is a normal distribution $\mathrm{N}\left(S_{i}, \sigma^{2}\right)$. The correlation of $X_{i}$ and $X_{j}$ is $\rho_{i j}=\rho(i, j=1,2, \cdots, n$; $i \neq j)$. The probability of times $X_{i}$ exceeds $X_{j}$ is

$$
\begin{aligned}
p_{i j} & =P\left(X_{i}>X_{j}\right)=P\left(X_{i}-X_{j}>0\right) \\
& =\frac{1}{\sqrt{2 \pi} \sigma_{i j}} \int_{0}^{\infty} \exp \left[-\frac{\left\{d_{i j}-\left(S_{i}-S_{j}\right)\right\}^{2}}{2 \sigma_{i j}{ }^{2}}\right] d d_{i j}
\end{aligned}
$$

where $d_{i j}=X_{i}-X_{j}$ and $\sigma_{i j}^{2}=2 \sigma^{2}(1-\rho)$.

Assigning the scale factor so that

$$
\sigma_{i j}^{2}=2 \sigma^{2}(1-\rho)=1 \text {, }
$$

we can rewrite the above equation

$$
p_{i j}=\frac{1}{\sqrt{2 \pi}} \int_{s_{j}-s_{i}}^{\infty} \exp \left\{-\frac{x}{2}\right\} d x .
$$

When we use the observed proportions $p_{i j}$, we can get $S_{j}-S_{i}$ from the normal distribution table. 


\section{Application}

Yamashita and Yonezawa ${ }^{[11]}$ revealed both the superiority of fuzzy ratings, which could incorporate the fuzziness and vagueness of a person's feelings in his/ her decision making, over crisp ratings in AHP, and the superiority of the crisp feedback, which exaggerates the superiority of only one specific alternative or exaggerates the differences among alternatives, over the final fuzzy weights from the fuzzy AHP. This seems to be because the fuzzy results might increase his/her hesitation about which alternative he/she should decide to choose, and the crisp feedback could help a decision maker make his/her decision. Yamashita $^{[14]}$ showed that it is very useful to apply Thurstone's paired comparison method to defuzzification of the final fuzzy weights from the fuzzy AHP.

This chapter exemplifies our method by using an example from Yamashita ${ }^{[14]}$. A male graduate student wanted to purchase one of the three kinds of cellular phones, $A_{1}, A_{2}$, and $A_{3}$, but he puzzled which product he should purchase. For supporting his decision making, the fuzzy AHP, which has the hierarchical structure with criteria "function," "design," "price," and "brand" as shown in Figure 3, was applied. He replied four numbers which corresponded to a fuzzy number as shown in Figure 2.

The results of the fuzzy AHP were presented with three kinds of forms :

1) final fuzzy weights which were fuzzy sets derived from the fuzzy AHP,

2) ranks obtained by Buckley's method ${ }^{[6]}$,

3) crisp scores obtained by Thurstone's paired comparison method which can defuzzificate the fuzzy weights and can exaggerate the the superiority of

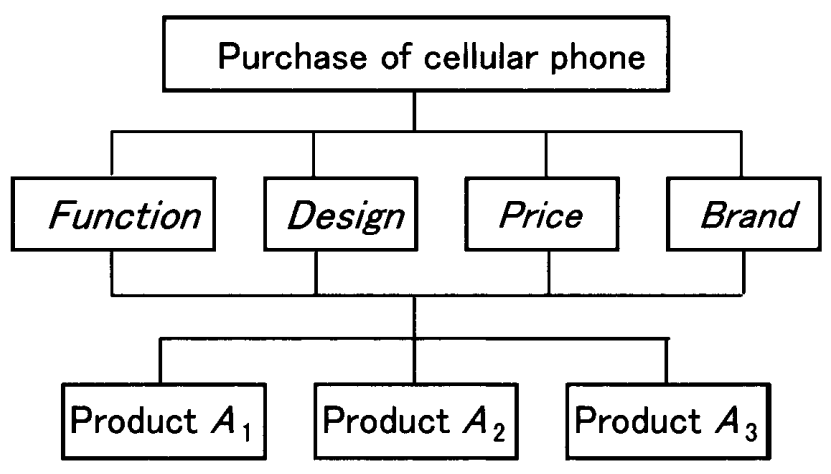

Figure 3 A hierarchy for AHP. one specific alternative and the differences among the alternatives.

Ranking of two fuzzy numbers was conducted according to the following method proposed by Buckley [6]. Let $\bar{M}$ and $\bar{N}$ be two fuzzy numbers with membership functions $\mu_{M}$ and $\mu_{N}$, as shown in Figure 4. Define

$$
\nu(\bar{M} \geq \bar{N})=\sup _{x \geq y}\left(\min \left(\mu_{M}(x), \mu_{N}(y)\right)\right) .
$$

It is assumed that $\bar{M}$ is greater than $\bar{N}$, written $\bar{M}$ $>\bar{N}$, if $\nu(\bar{M} \geq \bar{N})=1$ and $\nu(\bar{N} \geq \bar{M})<\theta$, where $\theta$ is some fixed positive fraction less than or equal to one. Moreover, if

$$
\min (\nu(\bar{M} \geq \bar{N}), \nu(\bar{N} \geq \bar{M})) \geq \theta
$$

then $\bar{M} \approx \bar{N}$. In this study, we set $\theta=0.7$.

The crisp scores were obtained as follows. We apply the paired comparison method by Thurstone ${ }^{[12]}$. ${ }^{[13]}$ to all the paired comparisons of the numbers each of which is one of the fuzzy numbers for $A_{i}, i=1,2,3$.

The results are shown in Figure 5. The fuzzy weights of the criteria were $(0.07 / 0.11,0.11 / 0.23)$, $(0.03 / 0.04,0.04 / 0.08),(0.20 / 0.29,0.31 / 0.47)$, and $(0.26 / 0.53,0.56 / 1.00)$ for function, design, price, and brand, respectively. Moreover, the final fuzzy weights for $A_{1}, A_{2}$, and $A_{3}$ were $(0.17 / 0.50,0.53 / 1.48),(0.15$ $/ 0.33,0.35 / 0.77)$, and $(0.07 / 0.15,0.16 / 0.40)$, respectively. The ranking by Buckley's method was $A_{3}<$ $A_{2} \approx A_{1}$. The weights obtained from the paired comparison method by Thurstone were $0.67,0.06,-0.73$ for $A_{1}, A_{2}$, and $A_{3}$, respectively. The paired comparison results generally exaggerate the superiority of only one alternative and the differences among alternatives. In this case, the superiority of $A_{1}$ was exaggerated as shown in Figure 5.

According to Yamashita ${ }^{[14]}$, the decision maker reported the superiority of the crisp feedback for both "effectiveness of supporting human decision making" and "goodness of fit to human feelings."

\section{Conclusion}

Although fuzzification techniques developed in the fuzzy set theory can represent the fuzziness in human feelings, a decision maker often wants a crisp feedback. Therefore, defuzzification techniques are also needed for supporting human decision making. In this paper, we have outlined our approach in which both fuzzification and defuzzification can be effectively used 


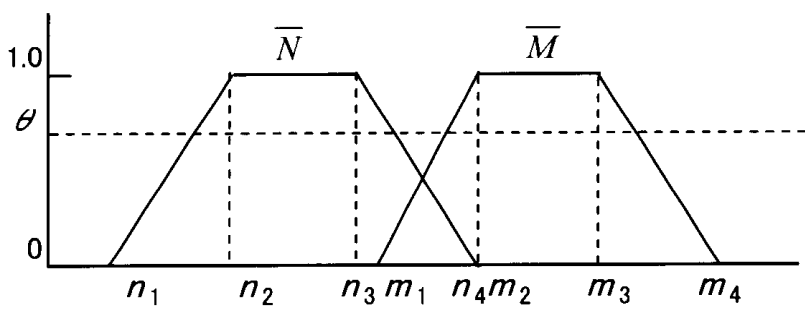

Figure 4 Example of membership functions of fuzzy numbers.

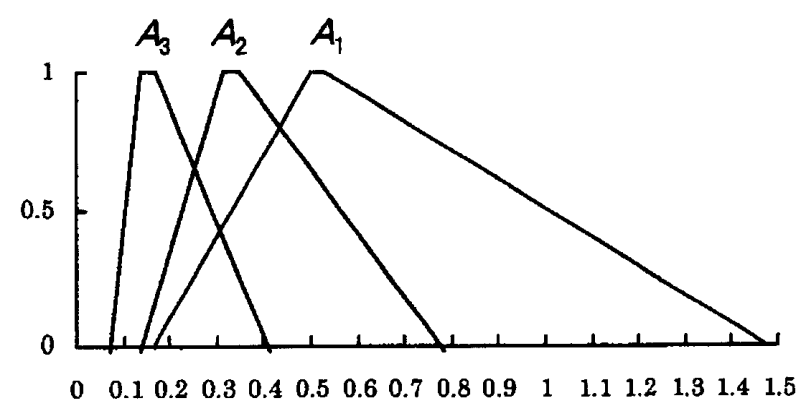

(a) Fuzzy weights

\section{$\mathrm{A} 3<\mathrm{A} 2 \approx \mathrm{A} 1$}

(b) Ranks

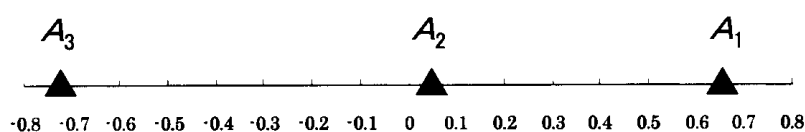

(c) Crisp scores

Figure 5 Three kinds of presentation forms showing the fuzzy AHP results ${ }^{[16]}$.

in fuzzy AHP. Our approach could provide a new way of looking at the effective use of fuzzy AHP for supporting human decision making.

\section{References}

[ 1] Yamashita, T. (1995). On the support system giving a feeling of satisfaction to a decision maker. Journal of Japan Society for Fuzzy Theory and Systems, 7, 44-51.

[2] Yamashita, T. (1997). On a support system for human decision making by the combination of fuzzy reasoning and fuzzy structural modeling. Fuzzy Sets and Systems, 87, 257-263.

[ 3 ] Saaty, T. L. (1977). A scaling method for priorities in hierarchical structures. Journal of Mathematical
Psychology, 15, 234-281.

[ 4 ] Saaty, T. L. (1980). The Analytic Hierarchy Process. McGraw-Hill, New York.

[5] Laarhoven, P. J. M. van and Pedrycz, W. (1983). A fuzzy extension of Saaty's priority theory, Fuzzy Sets and Systems, 11, 229-241.

[6] Buckley, J. J. (1985). Fuzzy hierarchical analysis. Fuzzy Sets and Systems, 17, 233-247.

[ 7 ] Zadeh, L. A. (1965) . Fuzzy sets. Information and Control, 8, 338-353.

[8] Hesketh, B., Pryor, R., and Gleitzman, M. (1989). Fuzzy logic : Toward measuring Gottfredson's concept of occupational social space. Journal of Counseling Psychology, 36, 103-109.

[9] Hesketh, B., Pryor, R., Gleitzman, M., and Hesketh, T. (1988). Practical applications and psychometric evaluation of a computerised fuzzy graphic rating scale. In T. Zetenyi (Ed.), Fuzzy Sets in Psychology, Elsevier Science Publishers B. V., pp.425-454.

[10] Hesketh, T., Pryor, R., and Hesketh, B. (1988). An application of a computerized fuzzy graphic rating scale to the psychological measurement of individual differences. International Journal of Man-Machine Studies, 29, 21-35.

[11] Yamashita, T., and Yonezawa, Y. (2004). Effective use of fuzzy AHP for supporting human decision making. Journal of Japan Society for Fuzzy Theory and Intelligent Informatics, 16, 26-32.

[12] Thurstone, L. L. (1927). A law of comparative judgment. Psychological Review, 35, 273-286.

[13] Mosteller, F. (1951). Remarks on the method of paired comparisons : I. The least squares solution assuming equal standard deviations and equal correlations. Psychometrika, 16, 3-9.

[14] Yamashita, T. (2004). Fuzzy ratings and crisp feedback in fuzzy AHP. Abstracts Proceedings of Joint 2nd International Confererence on Soft Computing and Intelligent Systems and 5th International Symposium on Advanced Intelligent Systems, 79.

[15] Osgood, C. E. (1952). The nature and measurement of meaning. Psychological Bulletin, 49, 197-237.

[16] Yamashita, T. (2006) . Fuzzy ratings and crisp feedback in fuzzy AHP for supporting human decision making. Journal of Advanced Computational Intelligence and Intelligent Informatics, 10 (in press)

(2005 年 10 月 31 日受付)

[Contact Address]

6-6 Asahigaoka, Hino, Tokyo 191-0065, Japan

Graduate School of Engineering

Tokyo Metropolitan University

Toshiyuki YAMASHITA

TEL : +81-42-585-8616

FAX : +81-42-583-5119

E-mail : tyamash@cc.tmit.ac.jp 
Information about Author

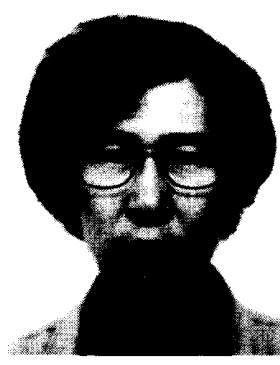

\section{Toshiyuki YAMASHITA [member]}

He obtained the Ph.D. in literature from Tokyo Metropolitan University in 1987. He is a professor, graduate school of engineering, Tokyo Metropolitan University. His research interests are in the area of human decision making, cognitive science, human factors, and cognitive engineering. $\mathrm{He}$ is a member of Japan Society for Fuzzy Theory and Intelligent Informatics, Japanese Psychological Association, Japan Ergonomics Society, and so forth. 